\title{
Two-stage Total Knee Arthroplasty in the Treatment of Advanced Knee Septic Arthritis: A Single-center Experience
}

\section{Hong Wang}

Lanzhou University Second Hospital

\section{Yuanjun Teng}

Lanzhou University Second Hospital

\section{Xingwen Wang}

Lanzhou University Second Hospital

\section{Shifeng Zhang}

Lanzhou University Second Hospital

\section{Tianen Xu}

Lanzhou University Second Hospital

\section{Hua Han}

Lanzhou University Second Hospital

\section{Yayi XIA ( $\nabla$ xiayayilzu@sina.com )}

Lanzhou University Second Hospital

\section{Research article}

Keywords: septic arthritis, knee infection, two-stage total knee arthroplasty, spacer Level of evidence IV

Posted Date: April 27th, 2021

DOl: https://doi.org/10.21203/rs.3.rs-433464/v1

License: (9) This work is licensed under a Creative Commons Attribution 4.0 International License. Read Full License 


\section{Abstract}

Background: Septic arthritis (SA) is defined as the joint inflammation secondary to bacterial infection with a potentially fatal condition, but with no established approach treatment currently. Two-stage primary total knee arthroplasty (TKA) with an antibiotic-laden cement spacer has been shown to be effective in treating SA of the knee in individual cases. We reported our cases to contribute to current information on two-stage TKA for the treatment of advanced SA in the knee joint

Methods: We retrospectively screened the clinical profiles of 11 patients who were suffering from SA in their knee joints and underwent two-stage TKA with an antibiotic-laden cement spacer. Information on inflammatory serological markers was summarised, and statistical comparisons of Hospital for Special Surgery (HSS) scores were performed. The degrees of the range of motion (ROM) in the pre-, interval- and post-operation periods were obtained, and the significance level was set at a $p$ value of $<0.05$.

Results: Infection in the knee joint was eliminated in all 11 patients with the two-stage TKA with an antibiotic-laden cement spacer. C-reactive protein levels returned to normal after $3.4 \pm 1.4$ weeks (range, 1-6 weeks), whereas erythrocyte sedimentation rate returned to normal levels after $16.3 \pm 3.0$ weeks (range, 13-23 weeks). The means of HSS scores and ROM degrees significantly increased after the first and second stages of surgery $(p<0.05)$. No difference in HSS score was observed in three periods between age groups, genders, microorganism culture results or comorbidities in our study.

Conclusions: Two-stage TKA appears to be an a reliable alternative for the treatment of advanced knee SA, providing an encouraging final clinical outcome in controlling infection, relieving clinical severity and improving the function of the keen joint.

\section{Background}

Septic arthritis (SA) is an emergency condition of joint infection, having an approximately two cases per 100,000 persons per year and the knee being the most commonly affected joint accounting for $40 \%-50 \%$ of reported cases in adults ${ }^{1-3}$. Patients with SA are often misdiagnosed with rheumatic disease and thus receive inadequate treatment ${ }^{4}$, which often leads to irreversible joint destruction and even case-fatality (i.e., $3 \%$ to $29 \%)^{5,6}$. Therefore, diagnosing this condition early and providing effective treatment immediately are indeed important ${ }^{1}$. The standard diagnosis of SA is based on the detection of bacteria in the synovial fluid through joint puncture. The primary therapy for SA includes the surgical interventions such as arthroscopic or open irrigation and debridement, in concerted with effective antibiotics utiliszation ${ }^{1}$. Arthroscopic surgery plays effective roles in SA treatment ${ }^{6,7}$, and open irrigation and debridement are usually considered for the patients with chronic infectious arthritis or persistent infection after arthroscopic surgery ${ }^{4,8}$. However, some cases show unsatisfactory recovery after such primary treatment, and eventually developed into non-salvageable destructive arthritis ${ }^{3}$. 
Some prospective pilot studies suggested that using implementing interventional articulate cement spacer in the problematic joints after total knee arthroplasty (TKA) is effective in controlling infection and improving functional outcomes $3,9-11$.

Previous studies were mostly case reports ${ }^{3,11-13}$, and evidences regarding the therapy aspect was limited. Therefore, this study aims assess the effificacy of application of a two-stage primary TKA to treat evolutive septic arthritis of knee joint, and contribute to existing literature and provide insights into this important topic for future research.

\section{Methods}

\section{Participants}

We retrospectively collected and investigated 11 eligible patients attending the Department of Orthopedic of Second Hospital of Lanzhou University in China from January 2012 to December 2018. The protocol was approved by the Medical Ethical Committee of the Second Hospital of Lanzhou University (Supplement), and informed written consent was obtained from participant/guardian. All the patients were diagnosed with SA on the basis of clinical features(purulent drainage, fever [temperature $>38^{\circ} \mathrm{C}$ ], and a painful, and restricted red joint), laboratory investigations(erythrocyte sedimentation rate [ESR] and levels of C-reactive protein [CRP] level), radiologic examination, and bacterial culture result of the joint fluid. The patients enrolled to this study fulfilled the following criteria of badly damaged knee joint, exfoliated cartilage and failure in controlling infection through arthroscopy, open debridement and appropriate antibiotic treatment. ${ }^{14}$ Two-stage arthroplasty was performed for the treatment of advanced knee SA consecutively (Figure 1). Surgery was conducted by a single surgical team. Deep infection was documented in all the 11 cases at the time of initial debridement. We did no apply any age or gender limitation. All the patients had their follow-up at our institution.

\section{Surgical technique and perioperative care}

\section{First stage}

In the first stage, a conventional medline knee skin incision was performed, followed by exposure of the joint through the parapatellar medial approach and complete removal of the synovial membrane, and infected and necrotic tissues. An intra-operative frozen section was obtained, and bacterial cultivation of the synovium and joint fluid was performed for the confirmation of the diagnosis and selection of susceptible antibiotic. Three biopsies were obtained during the first-stage procedure for postoperative pathological examination (Figure 2). Bone cutting was performed in preparation for primary TKA according to the size of the spacer, and all cuts were performed under extramedullary cutting guides for the prevention of intramedullary contamination. Measurements were obtained using the full-length images of the lower limbs as shown in Figure 3. The line from point A (i.e., the center of the femoral 
condyle) to point $D$ (i.e., the center of the femoral head) and the line from point $B$ to ponint $C$ (i.e., connecting line of the anterior superior iliac spine on both sides) were drawn for the cross point $E$, and the distance between point $B$ and point $E$ was measured and marked. During the operation, the line from points $A$ and $E$ were regarded as the virtual mechanical alignment of the lower limb. Finally, the cutting line of the distal femur was the vertical line of the line between points A and E. A spacer mold (Stage One; LINKXHamburg, Germany) was used in creating a dynamic antibiotic spacer from $80 \mathrm{gram}$ of Palacos R + G bone cement (Heraeus Medica, Hanau, Germany) mixed with gentamycin (4.8 gram) and vancomycin (8 gram). The cement was mixed without vacuum with a "poor mixing technique" for the enhancement of antibiotic elution ${ }^{15}$. Antibiotic-loaded bone cement was poured into the spacer block mold at the liquid stage, shaped as the prosthesis and taken out at the curing period. The antibiotic spacer was then applied to the bone surfaces with a poor bonding technique, in which blood was allowed to get between the spacer and bone in order that a certain degree of motion in the knee is possible before cement hardening, to facilitate subsequent removal. The femoral spacer block was inserted first and then an appropriate tibial spacer block adjusted with the extension gap was inserted in order that the limb was aligned and tissue tension was slightly relaxed for increasing post-operative range of motion (Figure 4). After numerous irrigations the soft tissues were closed with the use of a suction drain for less than 48 hours.

\section{Second stage}

Total knee arthroplasty was performed at least 12 weeks after laboratory inflammation markers had normalised and there was no further clinical evidence of infection (reddened and hyperthermic joint, drainage from the wound and unreasonable knee pain) was observed. Intraoperative selection cemented posterior stabilised prosthesis (Genesis II ${ }^{\text {TM }}$ total knee system, Smith \& Nephew, Memphis, TN, USA) was implanted, and patellar resurfacing was performed instead of patella replacement (Figure 5).

\section{Antibiotic treatment and perioperative care}

After the first stage of surgery, a two-week intravenous anti-biotherapy was administered according to bacterial culture, and sensitive results obtained after vancomycin was used for those without culture and sensitive results. Oral antibiotics (rifampicin + optimal antibiotics/levofloxacin) were administered for six weeks until two successive CRP results were normal and ESR results were below twice the value of the high threshold or had a continuous downtrend with no positive signs and symptoms. The CRP and ESR were monitored every 3 days in the first 2 weeks and every 2 weeks thereafter.

During the second stage of surgery, we applied $1 \mathrm{~g}$ of vancomycin for prophylaxis in the implementation of primary TKA surgical procedures ${ }^{16}$, and postoperatively vancomycin adminstration was continued until the wounds were dry. Then treatment was changed to oral rifampicin and levofloxacin for another 24 weeks ${ }^{17}$. Professional rehabilitation and functional training were implemented by rehabilitation 
physiatrists in our hospital after the two-stage surgery, and all patients were followed up every 3 months in the first year, and once per year thereafter.

\section{Statistical analysis}

Patient's files were screened by two authors for residual pain post-operative complications (including hematoma, seroma, blood transfusion, deep venous thrombosis, and revision surgery). Hospital for Special Surgery Knee (HSS) scores were obtained in pre-operation period (i.e., before the first-stage of TKA surgery), interval period (i.e., after the first-stage and before the second stage TKA surgery), and postoperation period (i.e., the latest follow-up) and were compared using analysis of variance (ANOVA) and post-hoc test of multiple group comparison. The effect of a two-stage operation was determined by comparing the degrees of pre-, interval- and post- knee range of motion (ROM) with paired t-tests. Twotailed independent t-test was applied to the comparisons between groups in terms of age (i.e., before or after middle age, following PubMed age filtration of 65 years old), gender, microorganism culture result (i.e., staphylococcus or negative) and comorbidities (i.e., have or not have). Data were reported as means \pm standard deviation (SD). All statistical analyses were performed using SPSS 22.0 (IBM Corp., Armonk, NY, USA). The level of significance was set at $p$ value of $<0.05$.

\section{Results}

Eleven patients comprising six males and five females were reported in this case-series (Table 1). Their mean age was $64.5 \pm 5.4$ years (range, $56-74$ years). All patients had invasive treatments, such as knee injection (i.e., in 7/11), arthroscopic debridement (i.e., in 6/11) and open debridement (i.e., in 9/11) previously. Four patients had comorbidities that might compromise immunity: one patient was diabetic, two patients were hypertensive and another had both. Regarding the micro-organism culture, six were positive for staphylococcus and the other five were negative, although utilized antibiotics were utilised variously.

\section{CRP and ESR level}

Infection was controlled successfully in all the patients by using the two-stage method for TKA. CRP level returned to normal level after $3.4 \pm 1.4$ weeks (range, $1-6$ weeks), whereas ESR returned to a normal level after $16.3 \pm 3.0$ weeks (range, $13-23$ weeks). The interval period was $14.4 \pm 4.2$ weeks (range, $10-24$ weeks) (Table 1).

\section{HSS scores}

The mean HSS scores were $38.5 \pm 16.4$ (range, 13-60) in the pre-operation period, $69.2 \pm 9.7$ (range, 5581 ) in the interval period and $85.2 \pm 5.5$ (range 74-92) (Table 1, Fig. 6a) in the post-operation. 
Comparisons of the HSS scores in these periods are shown in the Fig. 6a. The scores significantly increased $(p<0.01)$ after both the first stage $(p<0.01)$ and second stage $(p<0.01 ;$ Fig. $6 a)$. There was no significant difference identified of the HSS score in these three timings in age, gender, microorganism culture result and comorbidities.

\section{ROM}

The degree of ROM was increased $(p<0.05)$ after the first stage $(<0.05)$ and second stage $(p<0.05)$ (Fig. 6b), with a value of $54.5 \pm 31.8$ (range, $20-100$ ) in the pre-operation period, $76.8 \pm 20.9$ (range 35100 ) in the interval period and $90.5 \pm 16.7$, (range, 55-110) in the post- operation (Table 1). The average time of follow-up was $14.4 \pm 4.2$ months (range, $10-45$ months).

\section{Discussion}

Although therapeutic options for knee SA are limited, several studies applied two-stage primary TKA and attempted to improve its efficiency $2,3,11,18,19$, which encourage us to use the two-stage approach with a much shorter interval for the treatment of evolutive knee SA. In our study, elimination of infection, relief of symptoms and recovery of functions were fulfilled. Moreover, we reported experiences and endings that were the same as those reported in previous studies despite our small sample size. Therefore, two-stage TKA is a promising option for severe knee SA management.

Patients enrolled to this study were all diagnosed with knee SA, and fulfilled the criteria, particularly badly damaged knee joint, exfoliated cartilage and failure in controlling infection through arthroscopy , open debridement and appropriate antibiotic treatment. The operation mode was based from that of $B$ Hochreiter et al. ${ }^{10}$. Regarding the femoral cutting, we used a simple extramedullary guiding method in first stage to decrease the risk of surgery-related infections and improve function recovery. A previous study ${ }^{20}$ reported that valgus angles in $30 \%$ to $51 \%$ of patients who received TKA exceeded the valgus cut angle (i.e., 5-7 degrees) and are thus unsuitable for intramedullary guide tools and may result in the malalignment of the knee after TKA. Thus, in the first stage, we used a simple extramedullary guiding method in cutting the distal femur in all the 11 patients, which was equivalent to individualised valgus, and the alignment in these patients were appropriate. In addition, the extramedullary guiding method reduced the spread of infection and decreased the risk of bleeding and fat embolism. Further more, this extramedullary guiding method does not require $\mathrm{C}$-arm fluoroscopy for the femoral head during the operation and can thus reduce radiation injury.

The CRP levels of the 11 patients were returned to normal levels within 6 weeks after the first stage, although ESR decreasd more slowly. Previous studies suggested that biofilm formed in the spacer surface due to the long interval period, which casued infection recurrence. Our patients were suggested to undergo the second stage of surgery within 3 months for them to satisfy the following criteria: CRP test results that are more than twice the normal range, ESR halfly decreased and absence of symptoms of infection such as joint redness, fever, wound drainage and unreasonable knee pain. 
Infection were eliminated in the 11 patients, and their knee joint functions also returned to a level at which they were able to perform daily activities. However, patient 3 and 6 did not fully follow our advice to perform functional exercise after the first stage and thus had rigid knee joints surrounded by scar tissues. Thus, we had to perform the tibial tubercle osteotomy to expose the joint clearly and complete the operation in the second stage of surgery. Nevertheless, they had smaller ROM than other patients after surgery. We emphasised the advantages of functional exercises to them, including knee flexion and extension, lower limb muscle strength exercise and partial weight-bearing exercises, which accelerated their recovery post-operation. Knee joint function (i.e., HSS score) significantly increased after the twstage surgery. Therefore, function exercise should be performed in the entire process, especially within 1 month after each operation, because such activity affects the outcome of this surgery.

Previous studies on age and gender mainly focused on patients with osteoarthritis undergoing primary TKA and reported that females received primary TKA 3 years later than male patients and had lower knee function scores preoperatively and postoperatively 22 . Such findings demonstrated that the early initiation of treatment may enhance post-operative outcomes in women. Another study ${ }^{23}$ revealed that female gender, younger age and worsened preoperative pain predict increased risk of moderate to severe pain post-operatively in patients with primary and revision TKA.

However, information on factors influencing the functional outcomes of two-stage TKA for knee SA is indeed scant. We did comparisons in term of age, gender, micro-organism culture result and comorbidities but failed to identify differences.

However, similar to many previous studies $2,11,19,25$, our study has some limitations. First, our study was a retrospective study with a limited number of patients. The reason is that advanced knee arthritis combined with severe knee joint disability is uncommon and is difficult to treat. Second, we had no control group. Third, the average follow-up is not long enough, and we will continue to follow up further. Despite the small sample size, our study provided valuable information on this rare clinical challenge. We could concluded that the two-stage TKA with antibiotic-laden cement spacer is a potential option in the treatment for non-salvageable knee SA, and that extramedullary guiding method should be applied.

\section{Conclusion}

These results show that two-stage TKA is an a reliable alternative for the treatment of advanced knee SA, providing an encouraging final clinical outcome in term of infection control, clinical severity relief and knee joint function improvement.

\section{Abbreviations}

SA:septic arthritis; TKA:total knee arthroplasty; HSS: Hospital for Special Surgery; ROM:range of motion; CRP:C-reactive protein; ESR:erythrocyte sedimentation rate; 


\section{Declarations}

\section{Ethics approval and consent to participate}

The protocol for this study was approved by the Scientific Ethics Committee of the Second hospital of Lanzhou University. According to a retrospective design of the study, informed consent was waived and the authors had permission to access the data of patients in this study.

\section{Consent for publication}

All data generated or analyzed during this study are included in this published article.

\section{Availability of data and materials}

The datasets used and/or analysed during the current study are available from the corresponding author on reasonable request.

\section{Competing interests}

The authors declare that they have no competing interests.

\section{Funding}

This work was supported by The National Natural Science Foundation of China $(81874017,81960403$ and 82060413), Lanzhou Science and Technology Plan Program (2018-3-52), Cuiying Scientific and Technological Innovation Program of Lanzhou University Second Hospital (CY2017-QN12, CY2017ZD02), The Fundamental Research Funds for the Central Universities (Izujbky-2020-kb17), TCM inheritance and Innovation Platform construction project of Lanzhou University Second Hospital (TCMIPC-2020-05). The funding body made no other contributions to any aspects of this study.

\section{Authors' contributions}

HW wrote the manuscript and performed the data collection and

interpretation. YYX, HH and HW designed and performed the study. YJT performed the literature search and data collection, HW and XWW made

figures, and helped write the manuscript. HW, SFZ and TEX performed the literature search and data interpretation, and helped to write the manuscript. All authors read and approved the final manuscript. 


\section{Acknowledgments}

Not applicable.

\section{References}

1. John J. Ross, MD. Septic Arthritis of Native. Joints.Infect Dis Clin North Am. 2017 Jun;31(2):203-218.

2. Nazarian DG, de Jesus D, McGuigan F, Booth RE, Jr. A two-stage approach to primary knee arthroplasty in the infected arthritic knee. J Arthroplasty. 2003;18(7 Suppl 1):16-21.

3. Kirpalani PA, In Y, Choi NY, Koh HS, Kim JM, Han CW. Two-stage total knee arthroplasty for nonsalvageable septic arthritis in diabetes mellitus patients. Acta Orthop Belg. 2005;71(3):315-320.

4. Mathews CJ, Weston VC, Jones A, Field M, Coakley G. Bacterial septic arthritis in adults. Lancet . 2010;375(9717):846-855.

5. Balabaud L, Gaudias J, Boeri C, Jenny JY, Kehr P. Results of treatment of septic knee arthritis: a retrospective series of 40 cases. Knee Surg Sports Traumatol Arthrosc. 2007;15(4):387-392.

6. F. Aïm, J. Delambre, T. Bauer , P. Hardy.Efficacy of arthroscopic treatment for resolvinginfection in septic arthritis of native joints . Orthop Traumatol Surg Res .2015 Feb;101(1):61-4.

7. Mohammad Naghi Tahmasebi, MD, Arash Sharafat Vaziri, MD. et. al. Two-stage Total Knee Arthroplasty for Treatment of Surgical Failure of Septic Arthritis in Degenerative Knee Joints.Arch Bone Jt Surg. 2020 Jul; 8(4): 524-530.

8. Wang C, Lee YH, Siebold R. Recommendations for the management of septic arthritis after ACL reconstruction. Knee Surg Sports Traumatol Arthrosc : official journal of the ESSKA. 2014;22(9):21362144.

9. Bauer T, Lacoste S, Lhotellier L, Mamoudy P, Lortat-Jacob A, Hardy P. Arthroplasty following a septic arthritis history: a 53 cases series. Orthop Traumatol Surg Res. 2010;96(8):840-843.

10. Bae DK, Yoon KH, Kim HS, Song SJ. Total knee arthroplasty in stiff knees after previous infection. J Bone Joint Surg Br. 2005;87(3):333-336.

11. Hochreiter B, Strahm C, Behrend H. Short-interval two-stage approach to primary total knee arthroplasty for acutely septic osteoarthritic knees. Knee Surg Sports Traumatol Arthrosc. 2016;24(10):3115-3121.

12. Moyad TF. Two-stage arthroplasty for the treatment of chronic osteomyelitis after routine arthroscopic knee surgery. Orthopedics. 2013;36(1):e109-112.

13. Supreeth S, Al Ghanami S, Shanmugasundaram S, Al Rawi RS, Abdawani AR, Abdelmasih SR. Successful two-stage primary total knee arthroplasty for infective arthritis of the knee - our experience. J Clin Orthop Trauma. 2020;11(Suppl 5):S746-s751.

14. Eder L, Zisman D, Rozenbaum M, Rosner I. Clinical features and aetiology of septic arthritis in northern Israel. Rheumatology (Oxford). 2005;44(12):1559-1563. 
15. Lewis G. Properties of antibiotic-loaded acrylic bone cements for use in cemented arthroplasties: a state-of-the-art review. J Biomed Mater Res B Appl Biomater. 2009;89(2):558-574.

16. Anderson DJ, Podgorny K, Berríos-Torres SI, et al. Strategies to prevent surgical site infections in acute care hospitals: 2014 update. Infect Control Hosp Epidemiol. 2014;35 Suppl 2:S66-88.

17. Osmon DR, Berbari EF, Berendt AR, et al. Executive summary: diagnosis and management of prosthetic joint infection: clinical practice guidelines by the Infectious Diseases Society of America. Clin Infect Dis. 2013;56(1):1-10.

18. Shaikh AA, Ha CW, Park YG, Park YB. Two-stage approach to primary TKA in infected arthritic knees using intraoperatively molded articulating cement spacers. Clin Orthop Relat Res. 2014;472(7):22012207.

19. Seung-Suk Seo, Chang-Wan Kim, et al.Clinical outcomes of two-stage total knee arthroplasty using articulating cement spacer in patients with infected arthritic knee: A comparison with arthroscopic surgery .Knee.2020 Mar;27(2):444-450.

20. Bardakos N, Cil A, Thompson B, Stocks G. Mechanical axis cannot be restored in total knee arthroplasty with a fixed valgus resection angle: a radiographic study. J Arthroplasty. 2007;22(6 Suppl 2):85-89.

21. Parsley BS, Bertolusso R, Harrington M, Brekke A, Noble PC. Influence of gender on age of treatment with TKA and functional outcome. Clin Orthop Relat Res. 2010;468(7):1759-1764.

22. Singh JA, Gabriel S, Lewallen D. The impact of gender, age, and preoperative pain severity on pain after TKA. Clin Orthop Relat Res. 2008;466(11):2717-2723.

23. Aseem Arif Shaikh MS, Chul-Won Ha MD. Two-stage Approach to Primary TKA in Infected Arthritic Knees Using Intraoperatively Molded Articulating Cement Spacers. Clin Orthop Relat Res. (2014);472:2201-2207.

\section{Table}

Table 1 is not available in this version of the manuscript.

\section{Figures}




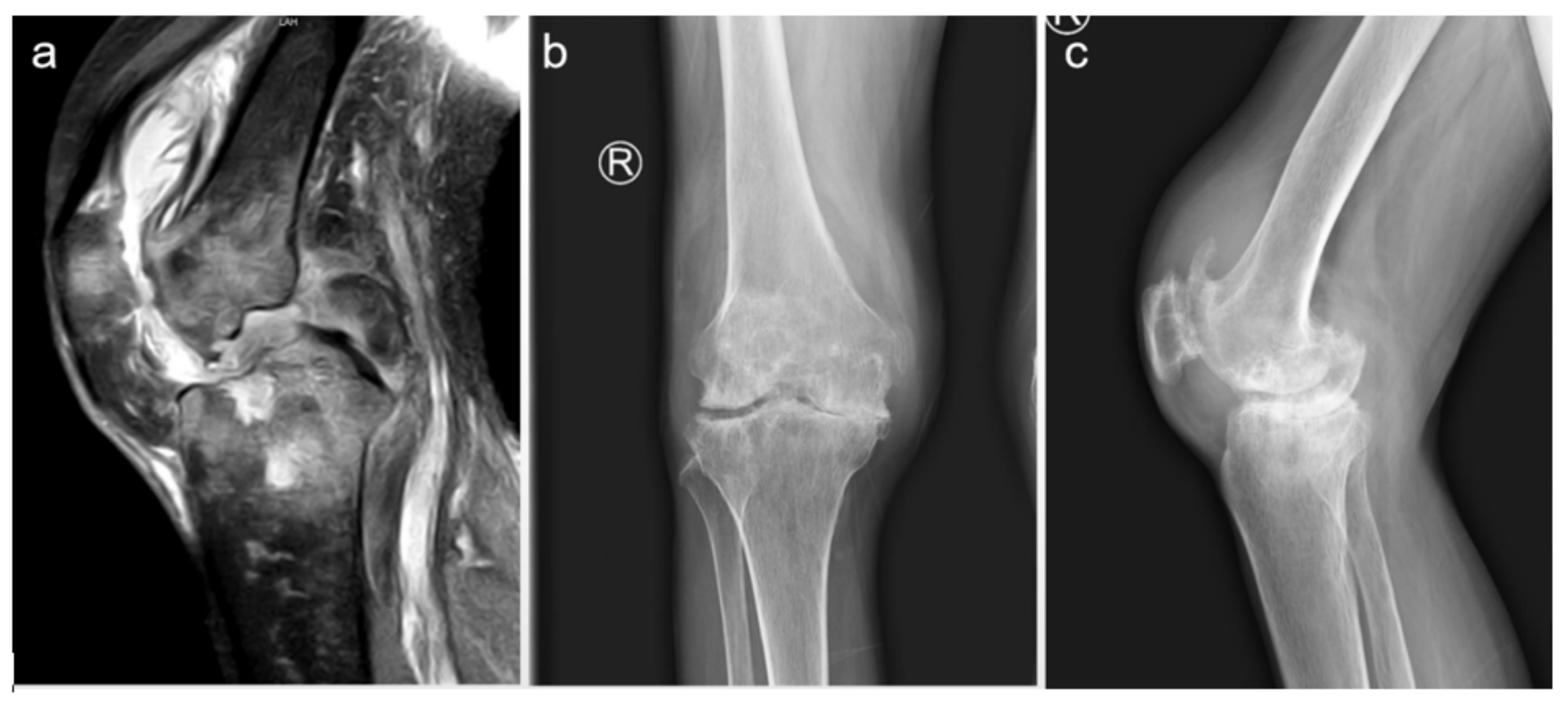

\section{Figure 1}

Images before surgery Images before surgery. (a) MRI PD-SPAIR image in Sagittal view; (b) X-ray image inanteroposterior (AP) view; (c) X-ray image in lateral view.
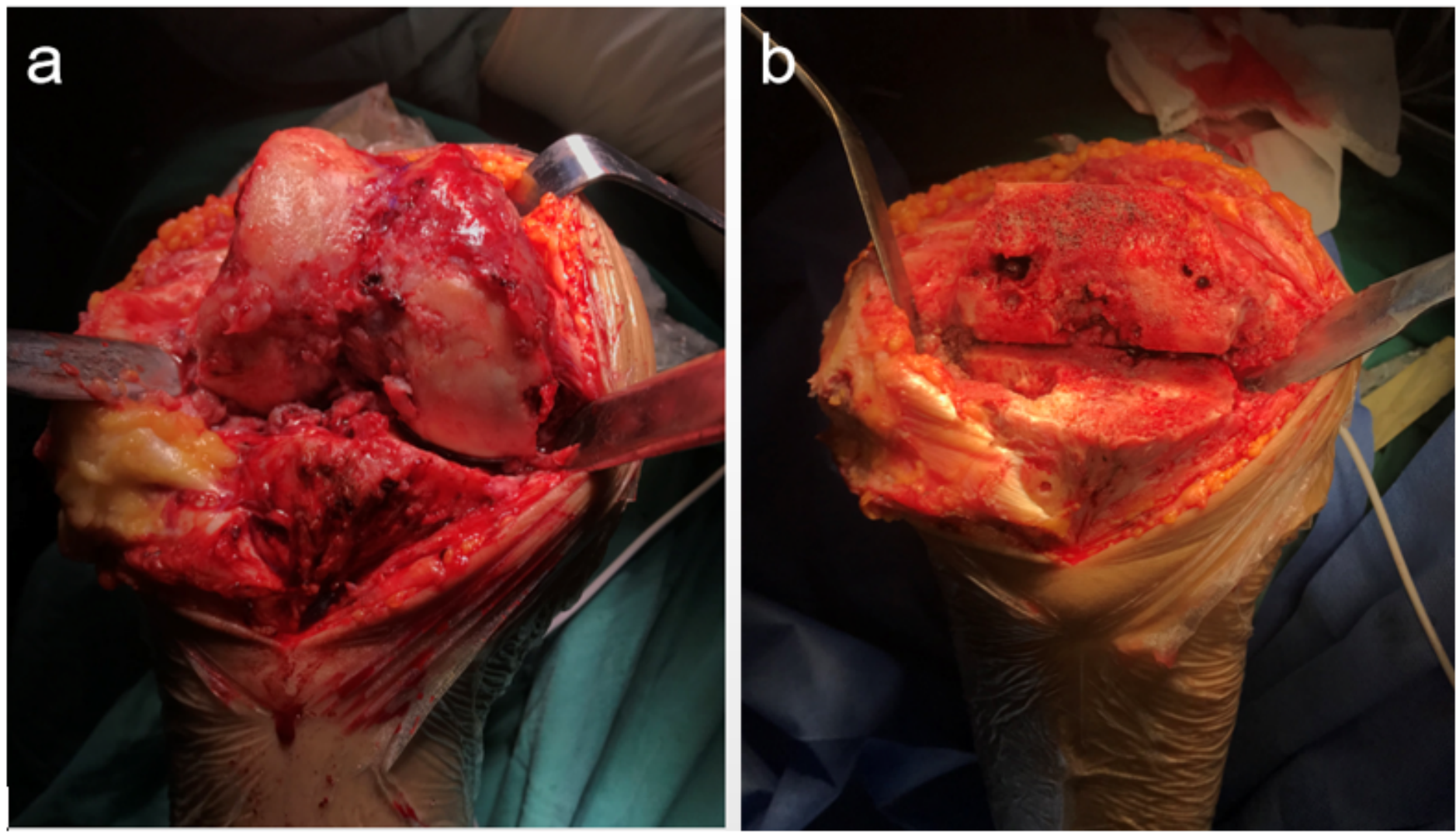

Figure 2 
Open surgery on knee joint in first stage A: intra-operative images of advanced septic arthritis of knee before debriding; B: image post debridement, extensive synovectomy and bone cutting.
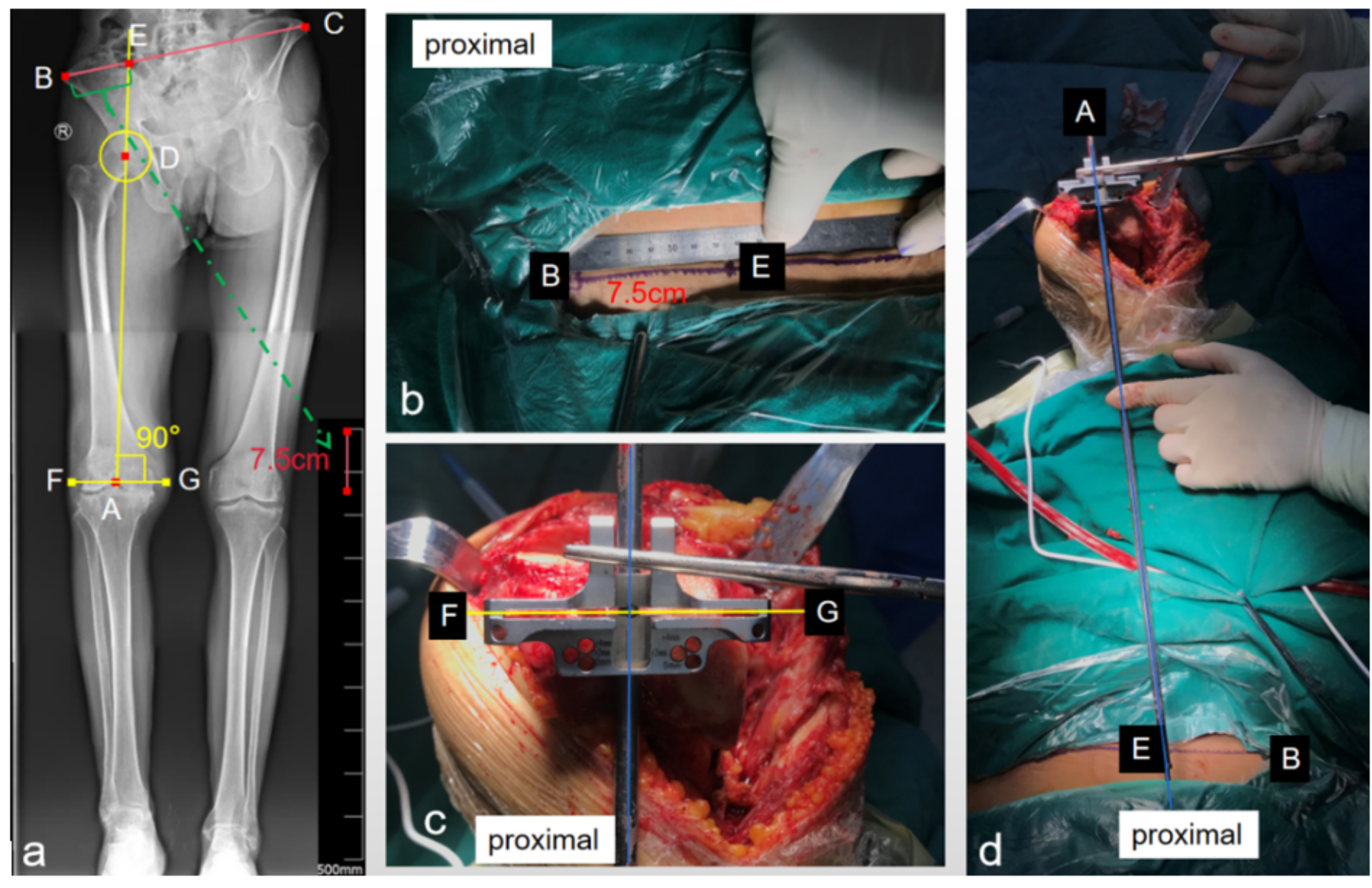

\section{Figure 3}

Extramedullary cutting guiding method Extramedullary cutting guiding method. (a) Measurements in extramedullary cutting guiding method; (b) Measurement of BE distance on body surface; (c) Final cutting line of distal femur; (d) Mechanical alignment on body surface. 

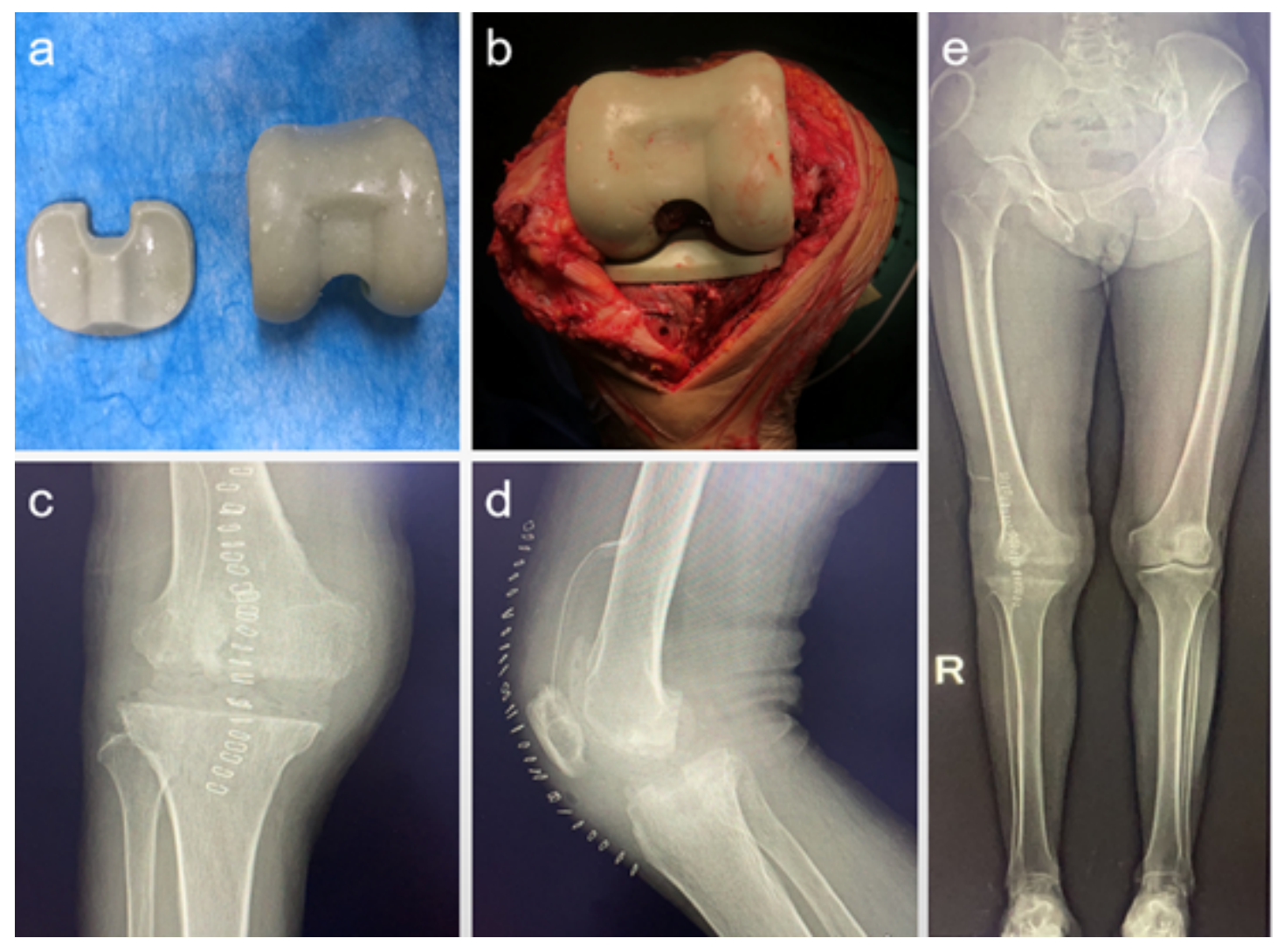

\section{Figure 4}

Images on spacer Images of spacer. (a) Dynamic antibiotic spacer created by a spacer mold; (b) Image of spacer when doing intra-operation; (c) and (d) Immediate post-operative images of AP and lateral views of the spacer; (e) Immediate post-operative full length image of AP view of the spacer. 

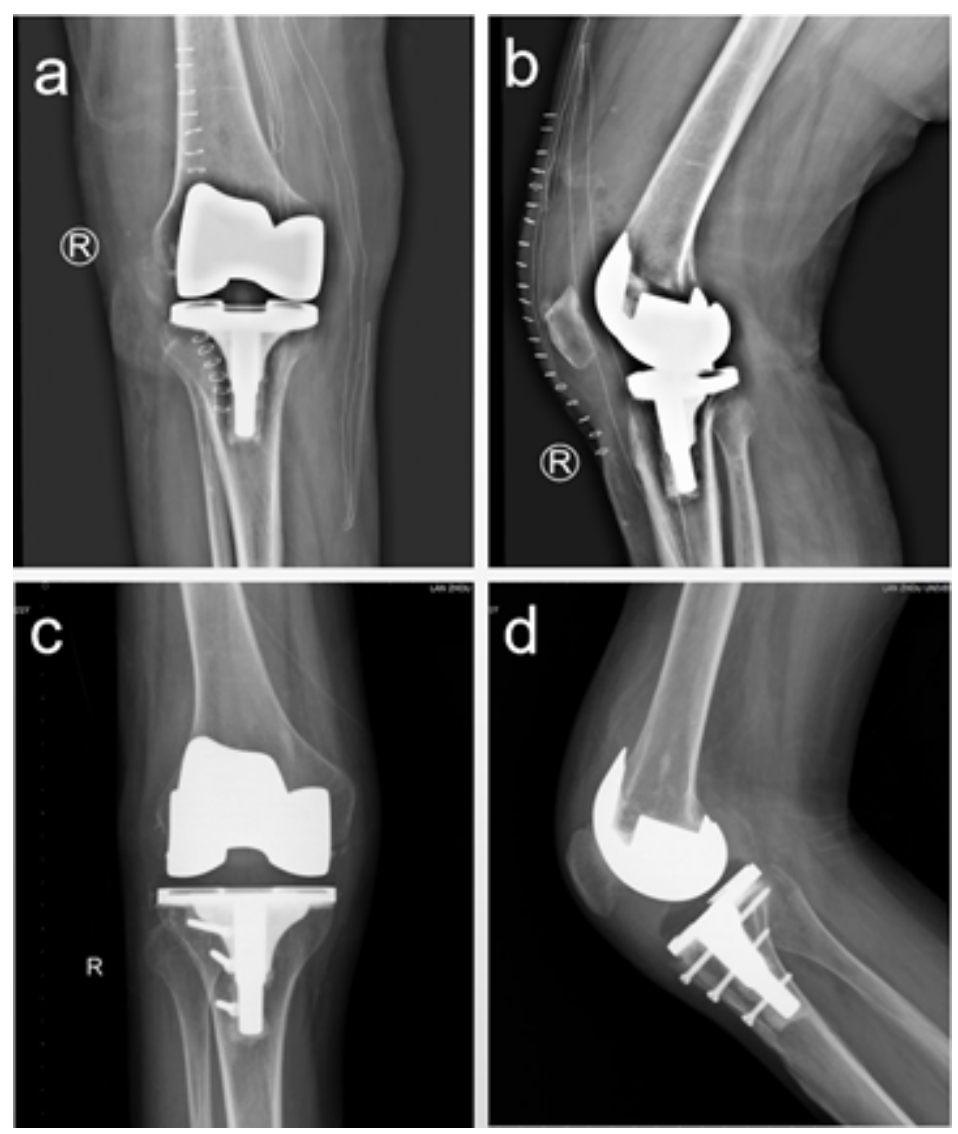

Figure 5

Knee joint after the second-stage surgery Knee joint after the second-stage surgery. (a) and (b) are postoperative X-ray after second stage operation; (c) and (d) are are postoperative X-ray after second stage operation with tibial tuberosity osteotomy.
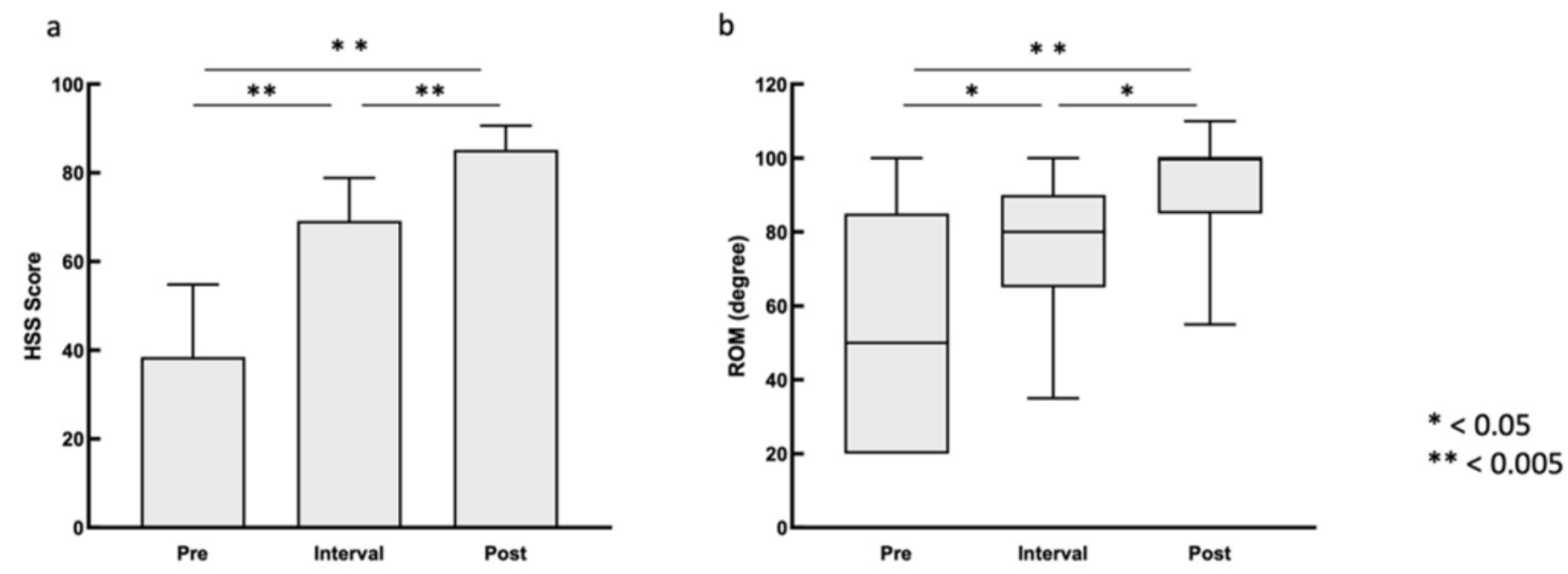

Figure 6 
Comparison of HSS and ROM Comparison of HSS and ROM. (a) Hospital for Special Surgery Knee score (HSS) among pre-operation, interval period and post-operation; (b) Range of motion (ROM) degree among pre-operation, interval period and post-operation. 